\title{
Management of extranodal lymphoma of the spine: a study of 30 patients
}

\author{
Shamsudini Hashi ${ }^{\ddagger 1}$, Courtney Rory Goodwin*,‡,2, Ali Karim Ahmed ${ }^{\ddagger 1}$ \& Daniel M Sciubba \\ ${ }^{1}$ Department of Neurosurgery, Johns Hopkins University School of Medicine, Baltimore, MD 21287, USA \\ ${ }^{2}$ Department of Neurosurgery, Duke University Medical Center, 200 Trent Dr., Durham, NC 27710, USA \\ *Author for correspondence: Tel.: +01 919684 7777; Fax: +01 919684 7937; rory.goodwin@duke.edu \\ ${ }_{\ddagger}^{\ddagger}$ Authors contributed equally
}

\section{Practice points}

- Spinal lymphoma is usually a late manifestation of systemic, disseminated disease.

- Few studies examine surgical outcomes or directly compare surgical and nonsurgical intervention for symptomatic relief.

- In this study, the medical records of 30 patients with malignant lymphoma of the spine were retrospectively reviewed.

- Non-surgical management is the primary treatment modality.

- Indications for surgery include emergent neurological deterioration, mechanical stabilization, refractoriness to medical management, or an open biopsy to obtain a pathological specimen.

Extranodallymphoma of the spine is often a late manifestation of systemic disease, and may result in symptoms of pain, neurologic compromise or spinal instability. Symptomatic relief is generally achieved by radiotherapy alone, but is not sufficient in addressing spinal instability. The indications for surgery remain controversial, but may be required for spinal stabilization, or refractory disease. Currently, there is a lack of studies that compare the indications and clinical outcomes of patients receiving surgical and nonsurgical management of spinal extranodal lymphoma. Medical records of 30 patients seen from March 2006 to August 2015, with histologically confirmed spinal lymphoma, were retrospectively reviewed. Demographic information, clinical factors, imaging, treatment and clinical outcomes were recorded. 19 patients were treated surgically and 11 nonsurgically (i.e., chemotherapy, radiation or combination). Surgery was performed for emergent neurological deterioration, mechanical stabilization, refractoriness to medical management or to perform an open biopsy for pathological diagnosis. Among those treated surgically, significantly fewer patients could carry on normal activities (KPS $<70$ ) at baseline, compared with those treated nonsurgically. However, there were no significant differences regarding pain medication use, functional status at 1 year, or mean survival ( 87.6 months) between groups. Surgery for extranodal lymphoma may be required in specific cases, resulting in favorable and similar outcomes compared with nonsurgical management.

First draft submitted: 25 September 2017; Accepted for publication: 12 February 2018; Published online: 30 April 2018

Keywords: adjuvant therapy $\bullet$ Hodgkin lymphoma $\bullet$ lymphoma $\bullet$ spinal instability neoplastic score $\bullet$ spine metastasis - surgery • survival • tumor

Lymphomas, accounting for nearly $5 \%$ of all adult cancers, are a group of heterogeneous blood cancers mainly arising in the lymph nodes [1]. Spinal lesions are estimated to occur in 5.8 and $6.5 \%$ of patients with Hodgkin and non-Hodgkin lymphoma, respectively [2]. Spinal lymphoma is usually a late manifestation of systemic, disseminated disease and malignant lymphoma causing epidural spinal cord compression is rare [3]. Similar to patients with other tumor histologies, patients with lymphoma involving the spinal column may present with debilitating pain, neurological compromise resulting from spinal cord compression or loss of spinal integrity. Given the radiosensitive nature of lymphoma and the current evidence supporting the use of radiotherapy alone for symptomatic relief (even in cases of progressive neurological decline), the role of surgical intervention in the management of spinal 
lymphoma remains unclear and controversial [4-8]. Contrary to the recommendation for radiation alone in the treatment of high-grade spinal cord compression due to lymphoma [6], evidence has demonstrated improved rates of neurologic recovery for patients undergoing urgent surgical decompression, compared with radiotherapy $[4,5]$. In the setting of neurologic dysfunction from spinal lymphoma, some have advocated that length of symptoms and nature of the neurologic deficit are important indications for surgery $[5,9]$. Furthermore, the patients with spinal lymphoma most commonly undergo surgical intervention in the setting of an unknown diagnosis, or for the stabilization of tumor-induced spinal instability $[10,11]$ - not possible with radiation.

The majority of studies on spinal lymphoma examine the medical management of patients, and few studies examine surgical outcomes or directly compare surgical and nonsurgical intervention for symptomatic relief [1216]. In this study, the medical records of 30 patients with malignant lymphoma of the spine were retrospectively reviewed. The aim of this study was to compare the treatment indications, management, clinical outcomes, and survival of patients who underwent surgical treatment for extranodal lymphoma of the spine, to those treated nonsurgically.

\section{Methods}

Study design \& subject inclusion

This was a retrospective study of patients with extranodal lymphoma of the spine and was approved by the Institutional Review Board, IRB number NA_00067508. A search of the institution's medical records identified 56 patients with histologically proven diagnosis of lymphoma involving the spinal column from March 2006 to August 2015. Following exclusion for patients with insufficient follow-up, 30 patients were included.

Patients were divided into two groups based on whether they underwent nonsurgical (chemotherapy and/or radiotherapy) treatment alone $(n=11,37 \%)$ or surgical intervention $(n=19,63 \%)$. Indications for operative and nonoperative management were based clinician discretion and the standard of best care [3-6,9,12,15], for patients treated at this single institution. For symptomatic treatment of spinal lymphoma, nonsurgical management was favored over surgery whenever possible. This included cases where the index spinal lesion was pathologically confirmed lymphoma, it was reasonable that symptomatic improvement could be achieved by more conservative options (i.e., radiation), and there was no spinal instability on imaging. The decision to operate was made at the discretion of the treating neurosurgeon, in the absence of nonsurgical options. Indications for surgical treatment included emergent neurologic deterioration, stabilization for overt or impending instability, or open biopsy to obtain a pathologic specimen. Emergent neurologic deterioration was an acute and significant decline in motor function where urgent surgical decompression was felt to be the best option to preserve motor function. An open biopsy was considered appropriate when computed tomography (CT)-guided needle biopsies were nondiagnostic, or in lesions where needle biopsies were not feasible. Patients with a very poor prognosis that presented with emergent neurologic deterioration, where surgery was the only option to preserve function, underwent a less invasive therapeutic surgical decompression. Nonsurgical management was preferred for nonemergent cases that met surgical criteria, with a very poor prognosis, where it was felt that surgery would not offer a sustainable benefit. Progressive weakness, or pain, refractory to other nonsurgical treatments, were also indications for surgery.

\section{Variables collected}

For each patient, epidemiological data such age, gender, comorbidities, tumor characteristics, presenting symptoms, spinal integrity, baseline status, treatment specifics, length of hospitalization, complications, and readmissions were retrospectively extracted from the electronic medical records. For those undergoing surgery, indication and intraoperative details such as procedure, approach, instrumentation, and perioperative complications were obtained from the surgeons' operative notes. Surgical complications: wound infections, wound dehiscence, hematoma, revision surgery; medical complications: sepsis, neutropenia, DVT/PE, comorbidities; length of postoperative hospital stay; and hospital readmissions were recorded. For those undergoing instrumentation, follow-up radiograph reports were reviewed at 1 month, 6 months and 1 year for complications and instrument integrity.

Ambulatory status, pain medication use and neurological function were graded by retrospective review of the medical record and recorded for the following time points: baseline, 1 month, 6 months and 1-year post-treatment. Neurological function and ambulatory status were assessed, at each time point, by Karnofsky Performance Status (KPS) and Frankel Grade. Pain medication use was graded from one (no medication) to five (intravenous narcotics). The Spinal Instability Neoplastic Score (SINS) [11], a standardized tool to grade neoplasm-related instability, was 
utilized to identify spinal instability. A baseline SINS score was calculated for each patient, utilizing pre-treatment radiographs.

Survival data was obtained from the PDS laboratory system, and confirmed by contacting the Social Security office. All survival data is reported as the time from the date of biopsy to last known living status as of 21 July 2016. The study was approved by our University's Institutional Review Board (Protocol NA_00067508).

\section{Statistical analysis}

Quantitative data are expressed as means or median for continuous, nonparametric variables and frequency for categorical variables. For intergroup comparison, Fisher's exact test was used for categorical data. A p-value of $<0.05$ is considered statistically significant. Survival statistics and Kaplan-Meier curves were calculated using SPSS Statistics 24 .

\section{Results}

\section{Patient demographics \& presenting characteristics}

Thirty patients with histologically proven spinal lymphoma were included in the current study. Out of those, $11(37 \%)$ were managed nonsurgically (Table 1). The median age at diagnosis for this group was 70 years with $73 \%$ of cases being male. The median length of follow-up was 16 months. Lymphoma types included low grade non-Hodgkin $(\mathrm{n}=5)$, high grade non-Hodgkin $(\mathrm{n}=5)$, as well as Hodgkin lymphoma $(\mathrm{n}=1)$. All lesions occurred in the thoracolumbar spine with $73 \%$ occurring in the lumbar segment. Upon review of pretreatment clinical and radiographic data, the median SINS was seven. Six (55\%) patients were graded to have a stable spine (SINS $\leq 6)$, four (36\%) had an indeterminate instability (SINS 7-12), and one (9\%) patient was grossly unstable (SINS $\geq 13$ ).

19 patients $(63 \%)$ underwent surgical intervention in this patient cohort (Table 2). With a median length of follow-up of 3 months, this group had a median age at diagnosis of 61 years with $58 \%$ of cases occurring in males. One patient (5\%) presented with Hodgkin lymphoma, four (21\%) with low grade non-Hodgkin lymphoma, and $14(74 \%)$ with aggressive non-Hodgkin lymphoma. $11(58 \%)$ of the lesions were located in the thoracic spine, five $(26 \%)$ in the lumbar spine, one $(5 \%)$ in the cervical spine, and two (11\%) spanned multiple spinal regions. According to the spinal instability neoplastic score criteria, the median SINS was a six. A total of $12(63 \%)$ of the cases were graded as stable, six (32\%) cases as indeterminate instability, and one (5\%) case as grossly unstable. Upon review of the operative notes, surgical indications included: rapidly declining neurological function in an emergent setting in the presence of an unknown diagnosis (26\%), progressive myelopathy unresponsive to medical treatment $(16 \%)$, pathological fracture requiring stabilization (16\%), and obtaining an open biopsy to establish a diagnosis $(42 \%)$ - half of them occurring after an undiagnostic CT-guided biopsy.

\section{Treatment}

Among patients undergoing nonsurgical intervention, five received chemotherapy alone. One patient, with a spinal lesion found incidentally, received radiation therapy alone. The remaining five patients in the nonsurgically managed cohort were treated with a combination of chemoradiation. One patient was refractory to combination therapy and later underwent an allogenic bone marrow transplant. Two patients underwent adjuvant vertebral cement augmentation to alleviate persistent back pain.

The surgical group consisted of individuals undergoing diagnostic/therapeutic laminectomy $(\mathrm{n}=10)$ or laminectomy plus fusion $(\mathrm{n}=9)$. Four patients in this cohort had failed previous treatment, two with combined chemoradiation and two with chemotherapy alone, with deteriorating neurological function. The postoperative treatment was known for 16 surgical patients, all of whom went on to receive adjuvant therapy. Postoperatively, nine patients received chemotherapy alone, one patient, determined to have low burden of disease systemically, received radiotherapy alone, and six patients received a combination therapy. For all applicable surgical and nonsurgical cases, radiotherapy was directed to the index spinal tumor.

\section{Surgical reconstruction \& instrumentation}

All surgical cases employed a posterior approach to the spine with two cases involving a combined posterior and anterior approach. Four thoracic cases had nerve roots transected. Three cases required revision surgery, one for persistent pain unresponsive to nonoperative measures and the others for dealing with postoperative complications. Nine patients underwent spinal fusion, seven undergoing just posterior instrumentation and two undergoing both anterior and posterior instrumentation. The median number of levels instrumented was five (range 3-8). All 
Table 1. Patient demographics and outcomes.

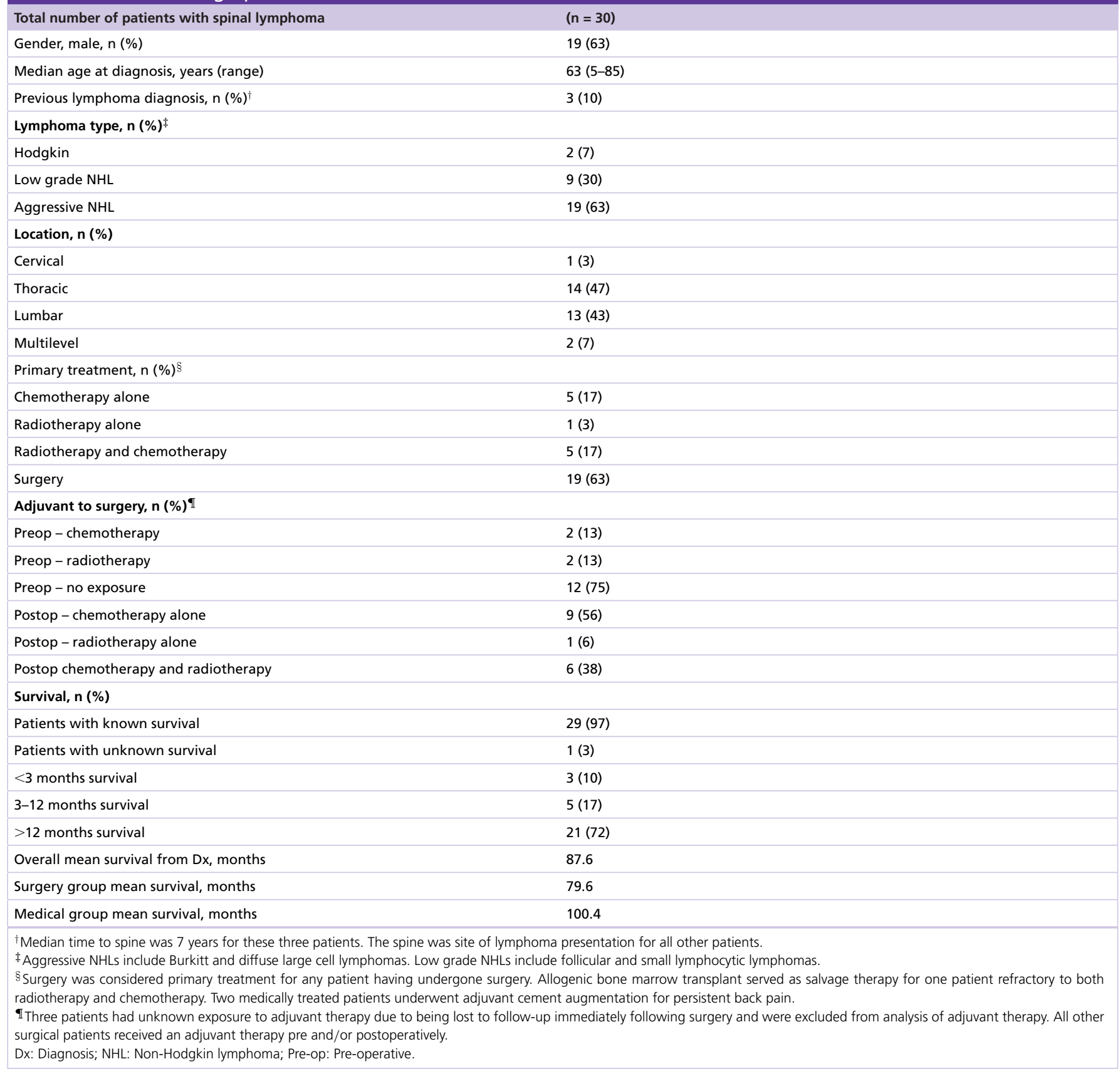

patients with follow-up radiographs at 1 month $(n=8), 6$ months $(n=2)$ and 1 year $(n=2)$ showed stable postoperative changes with intact hardware.

Six patients experienced a total of ten recorded postoperative complications. Three patients had poor wound healing leading to incisional dehiscence. Two of these cases occurred within 6 weeks (early) of surgery and the other 10 weeks (late) postoperatively. The late case was further complicated by a suspected enterococcus bacteremia requiring debridement and revision surgery. Two patients had a pulmonary embolus with one patient also developing a deep venous thrombosis and an epidural hematoma requiring evacuation. One patient developed postoperative ileus as well as a wound infection. 
Table 2. Functional outcomes.

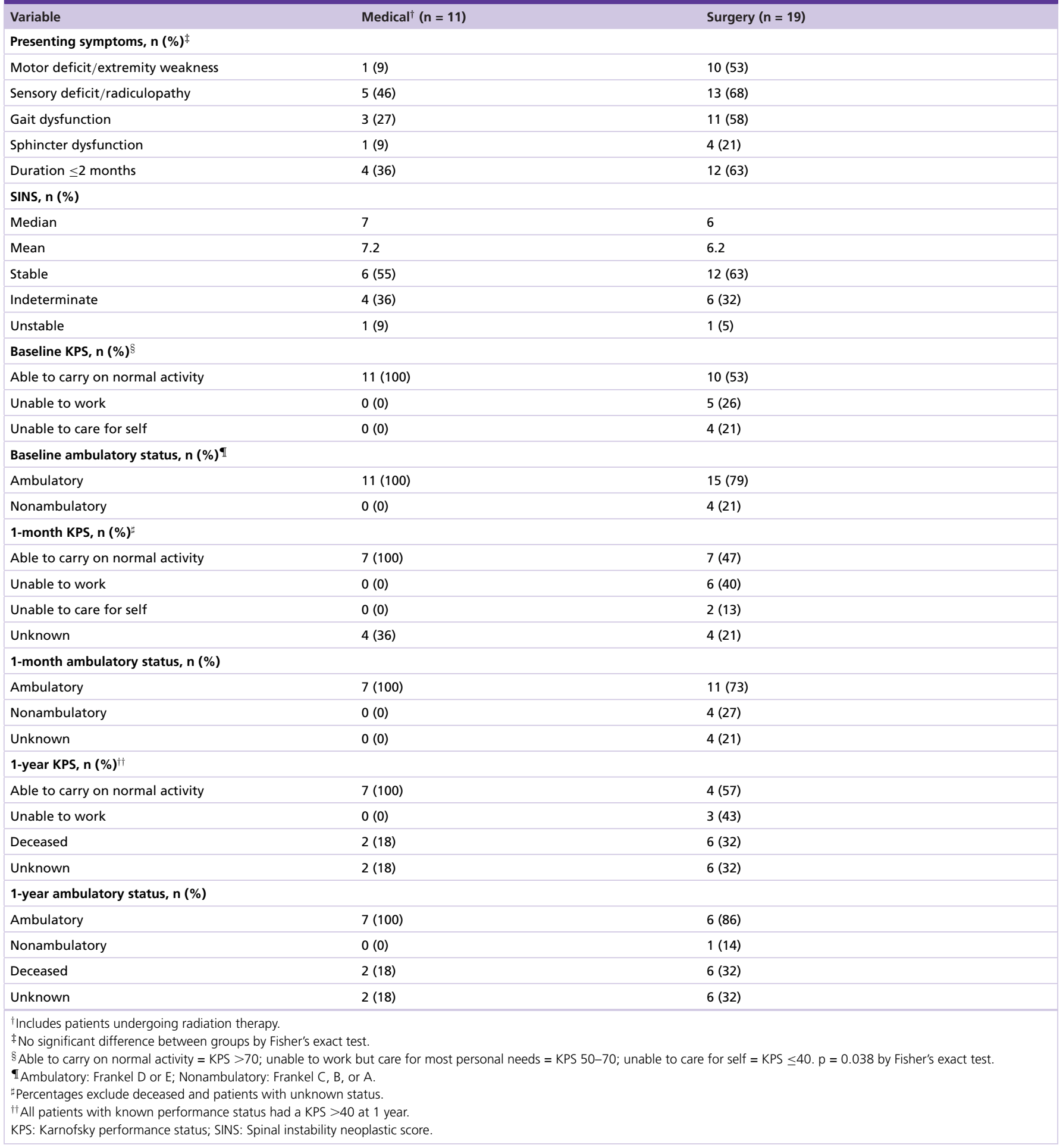

\section{Hospitalization}

Five nonsurgically treated patients were hospitalized upon initiation of treatment with a median length of stay of 4 days. Four patients accounted for a total of 11 known readmissions in the nonsurgical group. The median length of stay for these readmissions was 4.5 days. All patients undergoing surgical intervention required hospitalization with a median length of stay of 11 days (range 2-87 days). There was a total of 20 known readmissions among all surgical patients with a median length of stay of 7 days. Four patients experienced multiple readmissions. Medical 


\section{Table 3. Pain medication use.}

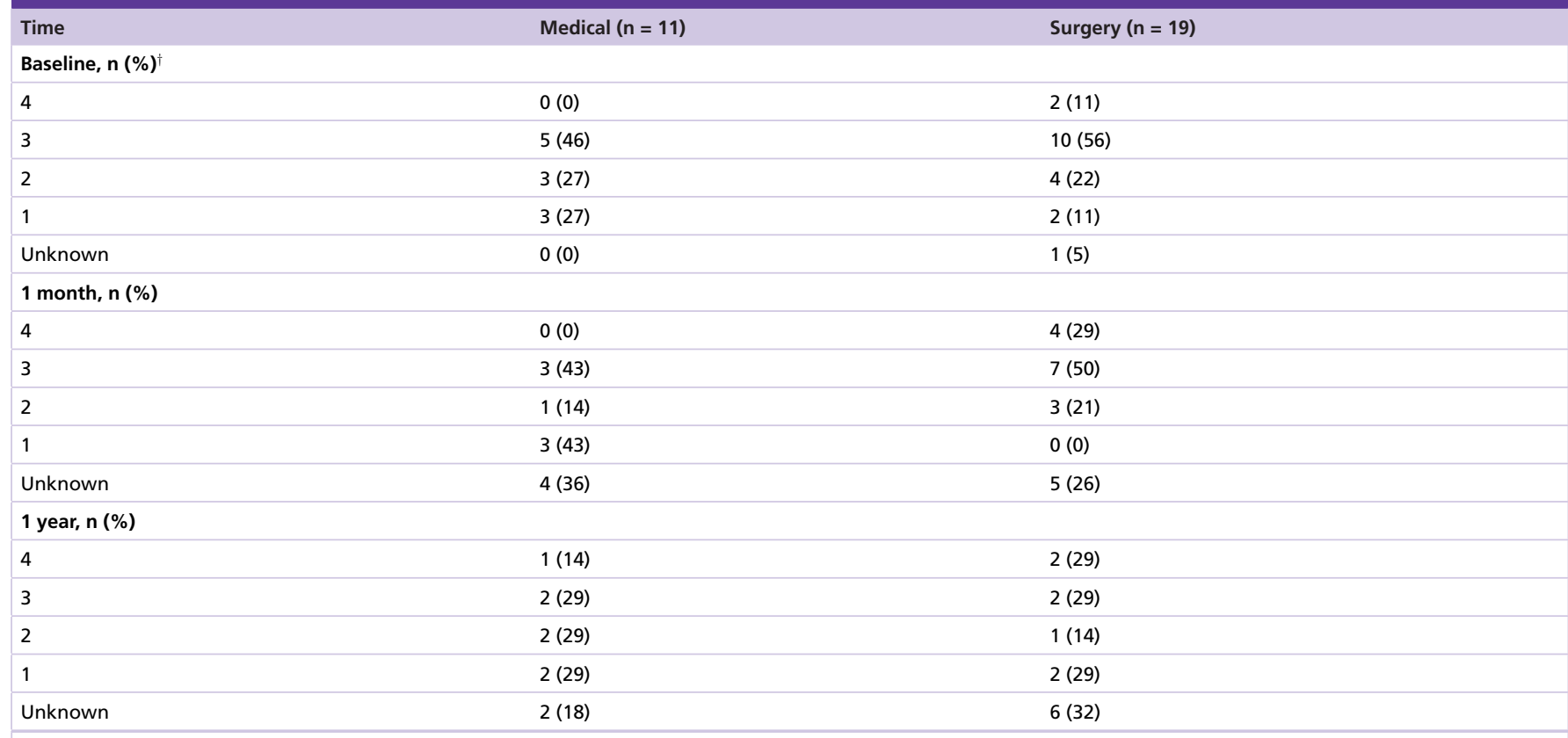

†Percentages exclude patients with unknown use.

Pain medication scores: 1: No medication; 2: NSAIDs, antiepileptics; 3: Codeine, hydromorphone, oxycodone; 4: Morphine SR/IR, fentanyl via transdermal patch, oxycodone SR/IR;

5: Intravenous narcotics.

\begin{tabular}{|c|c|c|}
\hline Complication & Early $\ddagger$ & Late \\
\hline Pulmonary embolus & 2 & 0 \\
\hline Wound dehiscence & 2 & 1 \\
\hline Wound infection & 1 & 1 \\
\hline Ileus & 1 & 0 \\
\hline Reoperation & 1 & 2 \\
\hline
\end{tabular}

complications leading to readmission included: neutropenic fever/sepsis, other infectious diseases, neurological dysfunction and uncontrollable spinal pain (Table 3).

\section{Functional \& pain outcomes}

Symptom presentation is represented in Tables $1 \& 2$. In summary, all patients were symptomatic at presentation, except for two nonsurgically managed patients who were found incidentally. The only significant differences between the treatment groups (Tables $4 \& 5$ ) by the Fischer's exact test were presence of motor deficits at presentation $(\mathrm{p}=0.023)$ and the baseline KPS $(\mathrm{p}=0.024)$. All nonsurgically treated patients were able to carry on normal activity (KPS $>70$ ) at baseline. $53 \%$ of patients undergoing surgery were able to carry on normal activity, 26\% were unable to work but care for most personal needs (KPS 50-70), and 21\% were unable to care for themselves (KPS $\leq 40)$ at baseline. For all patients with a baseline KPS $\leq 40(n=4)$, only one survived beyond 6 months. All living patients with complete follow-up data at 1 year $(\mathrm{n}=14)$ saw an improvement or a preservation of the KPS score, regardless of the treatment group.

All nonsurgically managed patients were ambulatory at all measured time points of this study. All nonambulatory (Frankel C or lower) patients $(n=4)$ underwent surgical intervention in an attempt to restore ambulatory function. Three of these patients died within 6 months of surgery. The other patient remains alive and nonambulatory 
Table 5. Re-admissions.

\begin{tabular}{|c|c|}
\hline Reason & Number of events \\
\hline Neutropenic fever/sepsis & 11 \\
\hline Other infection & 6 \\
\hline High-dose chemotherapy & 3 \\
\hline Uncontrollable pain & 3 \\
\hline Coagulopathy & 1 \\
\hline Neurological dysfunction & 2 \\
\hline Revision surgery & 1 \\
\hline Dyspnea & 1 \\
\hline Hypotension & 1 \\
\hline Fall & 1 \\
\hline Other $^{\dagger}$ & 1 \\
\hline
\end{tabular}

(Frankel C) 7 years postsurgery. In both treatment groups, all patients ambulatory (Frankel D or E) at presentation remained ambulatory at their last known follow-up.

As illustrated in Table 3 there is no significant difference in pain medication use at all time points between the two treatment groups. Prior to any intervention, 44 and $63 \%$ of nonsurgical and surgical patients, respectively, required narcotics for pain control. Three nonsurgical and two surgical patients reported no medication use at baseline. 1 year after the initiation of treatment, regular narcotic use was required by 57 and $43 \%$ of patients with known pain medication in the nonsurgical and surgical groups, respectively. A total of $36 \%$ of patients maintained their baseline pain medication score, 36\% reported an increase in their score, and $28 \%$ decreased the strength of medication needed for pain control 1 year after the start of treatment.

\section{Survival \& mortality}

Survival status as of 21 July 2016 was obtained for all patients, except for one international patient. On the last known inquiry, 20 patients were recorded as living. Out of all recorded deaths, $77 \%$ occurred in the surgical group. All patients dying within the first 3 months $(n=3)$ belonged to the surgical group, and presented with extensive lymphoma disseminated throughout the body in the soft tissue and bone. Five patients (17\%) died 3-12 months from the start of their treatment/date of surgery. The overall mean survival was 87.6 months with a mean survival of 79.6 and 100.4 months for the surgical and nonsurgical groups, respectively. There was no significant difference in the mean survival between treatment groups $(p=0.256)$, and between patients under and over the age of 65 $(\mathrm{p}=0.746)$ by a log rank test.

\section{Case illustrations}

Case 1

A 45-year-old man with a SINS of 15 and an unremarkable past medical history presented with a 5-month history of L5 distribution radiculopathy and progressively worsening 7/10 mechanical back pain relieved by sitting or lying down. Although independently ambulatory, he had limited mobility due to pain. He required Percocet for pain control. He was not found to have any other neurological deficits. CT and MRI imaging showed pathological destruction and loss of anterior column support of the L5 segment. CT-guided biopsy was consistent with diffuse large B-cell lymphoma (DLBCL). Initial staging images demonstrated disseminated disease. Given the mechanical instability, he underwent surgical stabilization and reconstruction from L3 to S2 through an anterior and a posterior approach in a staged fashion. His postoperative course was complicated by ileus and a wound infection necessitating a readmission. He was started on six cycles of R-CHOP 4 weeks postoperatively. On his last known visit, 3 months postoperatively, patient rated his pain as $0 / 10$ and only required the use of NSAIDs for pain control. Unfortunately, patient succumbed to his illness shortly afterward.

\section{Case 2}

A 56-year-old man, with a SINS of 5, presented to an outside hospital with saddle paresthesia, pain and weakness in his lower extremity. Patient was independently ambulatory, but unable to work due to 10/10 pain. MRI imaging 

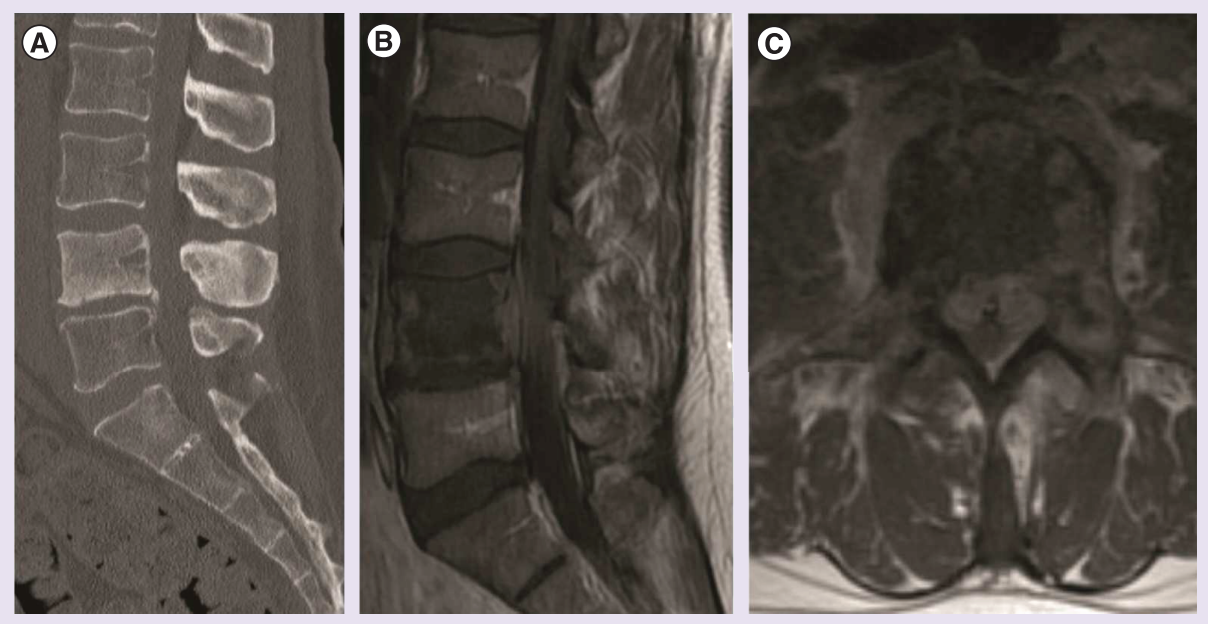

Figure 1. Preoperative representative imaging of a 56-year-old male with large B-cell lymphoma of the spine.(A) Sagittal CT without contrast. (B) Sagittal T1 MRI. (C) Axial T2 MRI.

CT: Computed tomography.

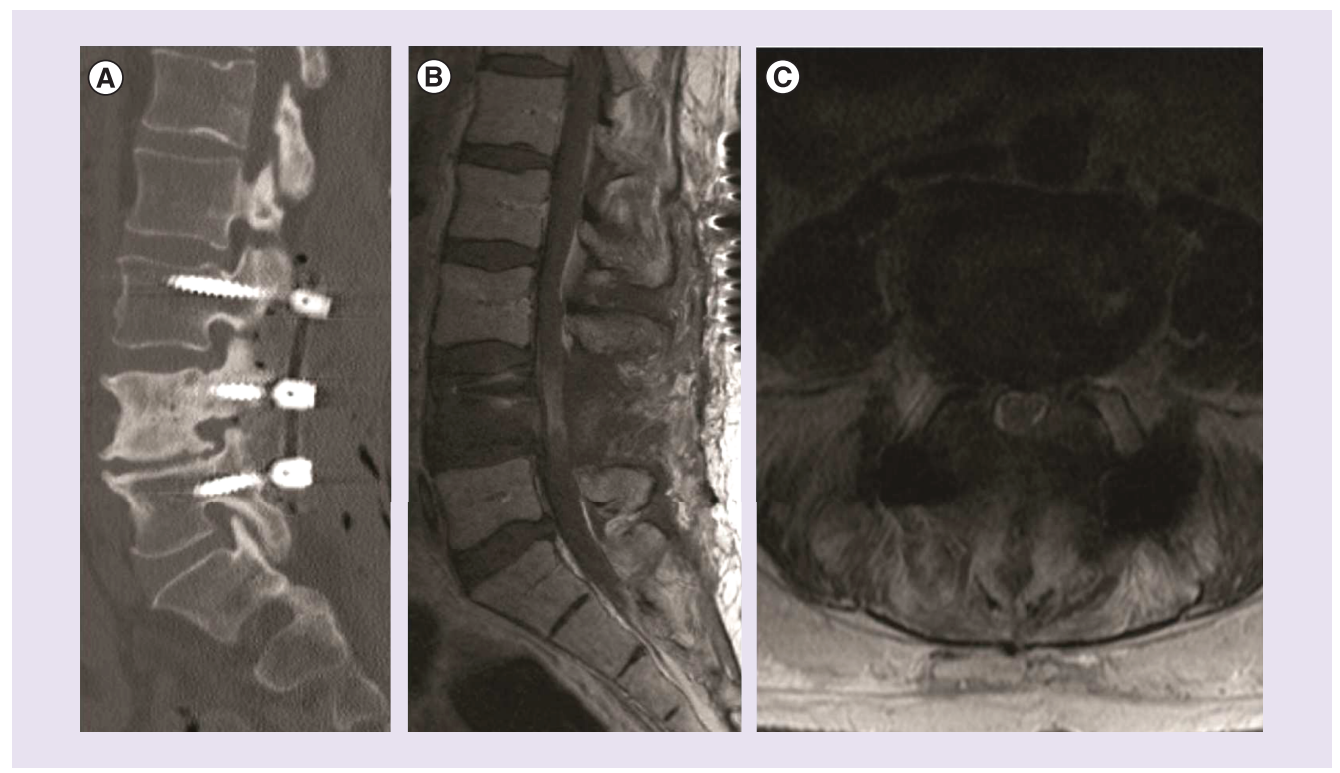

Figure 2. Postoperative representative imaging of a 56 -year-old male with large B-cell lymphoma of the spine.(A) Sagittal CT without contrast. (B) Sagittal T1 MRI. (C) Axial T2 MRI.

CT: Computed tomography.

showed severe spinal stenosis at L4 secondary to a suspected tumor mass (Figure 1). After three inconclusive needle biopsies, he was finally diagnosed with DLBCL. He was transferred to our facility for further management after showing no improvement with radiation therapy and high dose steroids. On admission, patient presented with urinary and fecal incontinence as well as bilateral lower extremity paralysis. He was presumed to have cauda equine syndrome, and underwent an emergent decompressive laminectomy of L4, total facetectomies of L4-5, and instrumentation with pedicle screws from L3 to L5 bilaterally secondary to medically refractory neurologic decline (Figure 2). He experienced a complicated postoperative course requiring a 3-month hospitalization. He attained complete remission with six cycles of R-CHOP and two doses of high-dose methotrexate. The patient is functionally paralyzed from the waist down, 7 years postoperatively, but is otherwise asymptomatic. 


\section{Case 3}

An 85 -year-old male, with an SINS of 15, presented with low back pain and difficulty walking. Initial MRI imaging showed metastatic disease involving the body of L2 with marked compression of the cauda equina. The patient was offered surgery for diagnostic and therapeutic purposes, but refused. A CT-guided biopsy was consistent with DLBCL. The patient was subsequently started on chemotherapy, but experienced progressive decline. The patient passed away 8 months after his initial presentation.

\section{Discussion}

Malignant lymphoma involving the spinal column is a rare occurrence, and due to the radiosensitivity of these tumors, radiotherapy is used as a first line treatment even in cases of neurological decline [6]. The precise role of surgery in the management of spinal lymphoma remains unclear, and mechanical instability remains the only agreed upon surgical indication [7]. Much of the current literature has focused on reporting outcomes for nonsurgical intervention, and there has been a limited exploration of the outcomes following surgical intervention. In this retrospective study, we present the epidemiological data, surgical indications, treatment and the comparative outcome data for 30 patients undergoing either surgical or nonsurgical intervention at a single major academic center over a 9-year period.

This study cohort was comprised of mostly male individuals in their sixth to seventh decade of life. Similar to that published in the literature, most extranodal lymphoma to the spine was non-Hodgkin lymphoma (93\% of cases), with a propensity for the thoracolumbar spine $[8,12,14]$.

The responsiveness of lymphomatous spinal lesions to adjuvant therapy is widely published in the literature [4$6,8,12-15]$. As such, both nonsurgically $(\mathrm{n}=11)$ and surgically $(\mathrm{n}=19)$ managed patients were exposed to systemic adjuvant therapy. Unlike most metastatic tumors of the spinal column, and malignant primary sarcomas, extranodal lymphoma of the spine responds to adjuvant chemoradiation within days [3,7]. Chemotherapy for DLBCL typically consists of R-CHOP (rituximab, cyclophosphamide, doxorubicin, vincristine, prednisolone) [7].

Highly radiosensitive tumors such as lymphoma are primarily managed with radiotherapy and adjuvant systemic therapy, except in cases of spinal instability [7]. The SINS was created by a panel of spine oncology experts for the purpose of establishing a standardized, easily applicable tool for grading spinal neoplastic related instability. The composite score was envisioned to aid in decisions pertaining to patient referral and patient selection for surgery [11]. The SINS is increasingly gaining clinical acceptance, and promising studies have shown its inter- and intra- observer reliability across the various medical and surgical disciplines [10,17-22]. However, this paradigm of selecting patient management based on tumor histology and SINS is dependent upon having an established diagnosis. Among the 19 surgical patients in this study group, only three had a biopsy proven diagnosis of lymphoma at the time of surgery. One of these patients, illustrated by Case 1, underwent a staged procedure for mechanical and structural spinal instability. The other two patients, illustrated by Case 2, progressed to near paraplegia under medical treatment, and surgery was used as a last attempt to preserve remaining neurological function. These two cases demonstrate the clinical decision making process when there is a known lymphoma diagnosis of the spine. The SINS is used as part of the initial guidelines for surgical consideration, but a careful evaluation of each individual's tumor response is essential for proper management. If a patient fails to show improvement or experiences further neurological decline under radiotherapy, surgical consultation should not be delayed in order to avoid irreversible neurological damage as seen in the above two patients. Both patients lost ambulatory function while under radiation therapy.

All other surgical cases (16) involved an unknown diagnosis at the time of surgery. Discounting emergency cases, a recurring theme was the inability to establish a diagnosis without an open biopsy. A significant number of patients (42\%) underwent surgery primarily to obtain a pathological sample with a noteworthy of cases occurring after multiple undiagnostic CT-guided biopsies. In the setting of an unknown diagnosis with progressive neurological deficits, the potential benefits may outweigh the risks of surgery. In such cases, surgery may provide immediate therapeutic benefit as well as the diagnosis needed for treatment initiation.

Patients with established surgical indications are needed to clearly evaluate the role of surgical intervention. In a recent study of surgical outcomes and prognostic factors for patients with DLBCL, Han et al. [5] found that surgical decompression independently improves recovery for those presenting with neurologic deficits secondary to epidural spinal cord compression. In a study comparing surgical and nonsurgical interventions for patients with DLBCL of the spine, Chang et al. [18] demonstrated 100\% recovery from baseline neurologic deficits following surgery, compared with only $20 \%$ in those treated nonsurgically. As such, acute neurologic deficit regardless of primary 
tumor etiology, is advocated by some $[5,9,18]$ as an indication for surgical decompression. In addition, surgery may be required to obtain an open biopsy for pathologic diagnosis and direct adjuvant therapies as previously published $[2,16]$. Consistent with these studies, indications for surgical treatment in the current work included emergent neurologic deterioration, stabilization for overt or impending instability, or open biopsy to obtain a pathologic specimen. Peng et al. [9] demonstrated no significant difference survival for patients treated surgically and nonsurgically for NHL of the spine; however the authors suggested that indications for surgery depend on individual priorities and nature of neurologic dysfunction.

Limitations of this study include the small sample size, retrospective study design, number of patients lacking an established diagnosis at the time of treatment, and the heterogeneity of the disease entity. The small sample size is mainly a function of the rarity of spinal lymphoma, usually a late manifestation of disseminated, systemic disease. This is evidenced by the fact that at our large tertiary academic center, we had a total of 56 histologically proven cases over a 9-year period. In addition to starting with a small patient cohort, some patients were able to receive their chemotherapy and/or radiotherapy locally increasing the number of patients lost to follow-up.

Lymphoma encompasses multiple disease entities, each with its own disease characteristics. However, due to the paucity of information available on the subject matter, the authors felt the need to include all subtypes of lymphoma.

\section{Conclusion}

Due to the sensitivity of lymphomatous tumors to chemoradiation, nonsurgical management is the primary treatment modality for this patient population. This study demonstrates indications for patients requiring surgical management for their disease burden to include: emergent neurological deterioration, mechanical stabilization of the spine, refractoriness to medical management, or most frequently performing an open biopsy to obtain a pathological specimen. The amount of patients who were able to carry on normal activities (KPS > 70), at baseline, was significantly lower for those that were treated surgically. No statistically significant difference was found in terms of pain medication use, preservation and/or restoration of neurological function, or survival between the two groups, this likely reflects the small cohort of patients that meet these indications in the surgical group. Future multicenter prospective studies on patients with these primary surgical indications are needed to provide further information.

\section{Financial \& competing interests disclosure}

General disclosures unrelated to the present work: CR Goodwin: UNCF Merck Postdoctoral Fellow and has received an award from the Burroughs Wellcome Fund and the Johns Hopkins Neurosurgery Pain Research Institute; AK Ahmed: NREF Medical Student Summer Research Fellowship; DM Sciubba: has consulting relationships with Medtronic, Globus, DePuy and Stryker. The authors have no other relevant affiliations or financial involvement with any organization or entity with a financial interest in or financial conflict with the subject matter or materials discussed in the manuscript apart from those disclosed.

No writing assistance was utilized in the production of this manuscript.

Informed consent disclosure

The authors state that they have obtained verbal and written informed consent from the patient/patients for the inclusion of their medical and treatment history within this case report.

Ethical conduct of research

The authors state that they have obtained appropriate institutional review board approval or have followed the principles outlined in the Declaration of Helsinki for all human or animal experimental investigations.

Open access

This work is licensed under the Attribution-NonCommercial-NoDerivatives 4.0 Unported License. To view a copy of this license, visit http://creativecommons.org/licenses/by-nc-nd/4.0/

\section{References}

1. Siegel RL, Miller KD, Jemal A. Cancer statistics, 2015. CA Cancer J. Clin. 65, 5-29 (2015)

2. Uehara M, Takahashi J, Hirabayashi H et al. Hodgkin's disease of the thoracic vertebrae. Spine J. 13, 59-63 (2013).

3. Koeller KK, Shih RY. Extranodal lymphoma of the central nervous system and spine. Radiol. Clin. North Am. 54, 649-671 (2016). 
4. Chang CM, Chen HC, Yang Y, Wang RC, Hwang WL, Teng CL. Surgical decompression improves recovery from neurological deficit and may provide a survival benefit in patients with diffuse large B-cell lymphoma-associated spinal cord compression: a case-series study. World J. Surg. Oncol. 11, 90 (2013).

5. Han S, Yang X, Jiang D et al. Surgical outcomes and prognostic factors in patients with diffuse large B-cell lymphoma-associated metastatic spinal cord compression. Spine 41, 943-948 (2016).

6. Kaloostian PE, Yurter A, Zadnik PL, Sciubba DM, Gokaslan ZL. Current paradigms for metastatic spinal disease: an evidence-based review. Ann. Surg. Oncol. 21, 248-262 (2013)

7. Laufer I, Rubin DG, Lis E et al. The NOMS Framework: approach to the treatment of spinal metastatic tumors. Oncologist 18, 744-751 (2013).

8. Love JG, Miller RH, Kernohan JW. Lymphomas of spinal epidural space. AMA Arch. Surg. 69, 66-76 (1954)

9. Peng X, Wan Y, Chen Y et al. Primary non-Hodgkin's lymphoma of the spine with neurologic compression treated by radiotherapy and chemotherapy alone or combined with surgical decompression. Oncol. Rep. 21, 1269-1275 (2009).

10. Fisher CG, Schouten R, Versteeg AL et al. Reliability of the spinal instability neoplastic score (SINS) among radiation oncologists: an assessment of instability secondary to spinal metastases. Radiat. Oncol. 9, 69 (2014).

11. Fourney DR, Frangou EM, Ryken TC et al. Spinal instability neoplastic score: an analysis of reliability and validity from the Spine Oncology Study Group. J. Clin. Oncol. 29, 3072-3077 (2011).

12. Avilés A, Fernández R, González JL et al. Spinal cord compression as a primary manifestation of aggressive malignant lymphomas: long-term analysis of treatments with radiotherapy, chemotherapy or combined therapy. Leuk. Lymphoma 43, 355-359 (2002).

13. Correale J, Monteverde DA, Bueri JA, Reich EG. Peripheral nervous system and spinal cord involvement in lymphoma. Acta Neurol. Scand. 83, 45-51 (1991).

14. Friedman M, Kim TH, Panahon AM. Spinal cord compression in malignant lymphoma treatment and results. Cancer 37, 1485-1491 (1976).

15. Haddad P, Thaell JF, Kiely JM, Harrison EG, Miller RH. Lymphoma of the spinal extradural space. Cancer 38, 1862-1866 (1976)

16. Thomas AG, Vaidhyanath R, Kirke R, Rajesh A. Extranodal lymphoma from head to toe: part 1, the head and spine. AJR Am. J. Roentgenol. 197, 350-356 (2011).

17. Arana E, Kovacs FM, Royuela A, Asenjo B, Pérez-Ramírez Ú, Zamora J. Spine instability neoplastic score: agreement across different medical and surgical specialties. Spine J. 16, 591-599 (2016).

18. Campos M, Urrutia J, Zamora $\mathrm{T}$ et al. The spine instability neoplastic score: an independent reliability and reproducibility analysis. Spine J. 14, 1466-1469 (2014).

19. Fisher CG, Versteeg AL, Schouten R et al. Reliability of the spinal instability neoplastic scale among radiologists: an assessment of instability secondary to spinal metastases. Am. J. Roentgenol. 203, 869-874 (2014)

20. Huisman M, van der Velden JM, van Vulpen M et al. Spinal instability as defined by the spinal instability neoplastic score is associated with radiotherapy failure in metastatic spinal disease. Spine J. 14, 2835-2840 (2014).

21. Ivanishvili Z, Fourney DR. Incorporating the spine instability neoplastic score into a treatment strategy for spinal metastasis: LMNOP. Global Spine J. 4, 129-136 (2014).

22. Versteeg AL, van der Velden JM, Verkooijen HM et al. The effect of introducing the spinal instability neoplastic score in routine clinical practice for patients with spinal metastases. Oncologist 21, 95-101 (2016). 
